\title{
Rapid screening and visual tracing of melamine in soybean meal by NIR microscopy imaging
}

\author{
Zengling Yang*, Chengte Wang*, Lujia Han*,†, Jing Li* and Xian Liu* \\ *College of Engineering, China Agricultural University \\ Beijing 100083, P. R. China \\ ${ }^{\dagger}$ State Key Laboratory of Animal Nutrition \\ Beijing 100091, P. R. China \\ thanlj@cau.edu.cn
}

Received 1 July 2013

Accepted 16 October 2013

Published 2 December 2013

\begin{abstract}
Near infrared microscopy imaging offers the opportunity to explore not only what kinds of chemical species are present at micro-scale level but also where the chemical species would be present. By revealing the spectral and spatial information, the technique can identify and localize any interested component. This study investigates the feasibility of using Near infrared microscopy imaging to detect melamine in soybean meal. The results showed that $6805 \mathrm{~cm}^{-1}$ is very sensitive for melamine but not for soybean meal, so can be used for univariate analysis. Single wavelength image and peak integration image at $6805 \mathrm{~cm}^{-1}$ are simple and effective methods to detect the melamine in soybean meal. Furthermore, Principal Component Analysis is applied to detect the melamine in soybean meal.
\end{abstract}

Keywords: Near infrared microscopy imaging (NIRM imaging); soybean meal; melamine; univariate analysis; PCA.

\section{Introduction}

Consumers and governments are pushing for safer food and feed management systems. In this system, detecting techniques are the main technical support. The application of near infrared reflectance spectroscopy (NIRS) in feed quality and safety control is widely accepted, which includes chemical composition, and adulteration or contamination ingredients of raw material and compound feeding stuff. $^{1,2}$ NIRS provides integrated information of a bulk sample at macro-scale level with resolutions depending on the spot size generated by the beam of light.

Near infrared microscopy (NIRM) is able to collect the spectrum of individual particles (the minimum spot size is a few micrometer) from samples. The NIRM instrument includes a classical NIR spectrometer coupled with an optical microscopy in which the optics has been adapted to NIR radiation. A viewing system magnifies the visible light image

This is an Open Access article published by World Scientific Publishing Company. It is distributed under the terms of the Creative Commons Attribution 3.0 (CC-BY) License. Further distribution of this work is permitted, provided the original work is properly cited. 
of the sample, allowing the user to visualize it and position the infrared beam on the interested sample area using a motorized stage. Near infrared microscopy imaging (NIRM imaging) is obtained and developed by line imaging with an automatic moving stage. The technique offers the opportunity to explore not only what chemical species are present at a micro-scale level but also the size of the cluster of the ingredient in the formulation and how it combines to other components. ${ }^{3}$

In the past decade, NIRM imaging has become a powerful analytical tool in quality control and troubleshooting of pharmaceutical products ${ }^{3-8}$ and in the detection of contamination and defects in agro-food products. ${ }^{9-15}$ For feed products, there are reported investigations on the feasibility of using NIRM to detect, indentify and quantify processed animal by-products. ${ }^{16-20}$

With NIRM imaging, hundreds or thousands of spectra (including tens or hundreds of variables) need to be collected for each sample. This compares to an average spectrum with classical spectroscopic instrumentation. ${ }^{10}$ It is a challenge to handle, extract, and exploit such a large amount of data. By revealing information that is both spectral and spatial, the technique can identify and localize compounds. ${ }^{21}$

Soybean meal is one of the most important protein feed raw materials because it offers a complete protein profile. Over past 10 years, the price of soybean meal has risen by three times (http://faostat3.fao.org/home/index.html). The price of soybean meal is higher with higher protein content. In recent cases, soybean meal adulterated with melamine (1,3,5-triazine-2,4,6-triamine) had been found to boost the protein content. ${ }^{22}$ The adulteration of melamine in feed is a great concern for feed safety. There were many analytical methods for detecting melamine. ${ }^{23}$ Vibrational spectroscopy, such as Raman spectrometry, near- or mid- infrared spectroscopy, was a good way to detect melamine because of their advantages compared to other methods. ${ }^{24,25}$ Comparing with these techniques, NIRM imaging have advantages to detect the adulterant. First, NIRM imaging can provide the spectral information of sample at a micro-scale level, so it is easy to exactly find the target spectral characteristic. Second, NIRM imaging can provide the spatial information of sample at a micro-scale level, so it can confirm the position of target inside the testing sample. The aim of this paper is to investigate the feasibility of using NIRM imaging to rapidly screen and visually trace melamine in soybean meal. Different data processing techniques are used for extracting relevant information.

\section{Material and Methods}

\subsection{Instrument}

Samples were analyzed on a line imaging system (Spotlight400, Perkin Elmer), whereby 16 spectra are acquired simultaneously from a line detector. The detector of this instrument is a mercury cadmium telluride ( $\mathrm{HgCdTe}$ or $\mathrm{MCT})$. The spatial resolution of scanning is $25 \mu \mathrm{m} \times 25 \mu \mathrm{m}$ and spectral resolution is $32 \mathrm{~cm}^{-1}$. Ratio spectra were collected using a Teflon Spectralon (Spectralon $\mathbb{R}$ Targets, Labsphere) as the reflectance standard. Each spectrum is the average of four scans across the wavenumber range $7800-4000 \mathrm{~cm}^{-1}$.

\subsection{Experiment}

Soybean meal was collected from feed manufacture and ground to $1 \mathrm{~mm}$. Melamine was analytical reagent $(\geq 99.5 \%)$. Two sets of samples were prepared to investigate the method of screening and tracing melamine in soybean meal.

Set A: There were five different arrangement statuses for the particles from soybean meal and melamine if the monolayer of mixture of soybean meal and melamine was laid on Teflon Spectralon surface. They were a melamine particle placed on top of a soybean meal particle, a melamine particle placed under a soybean meal particle, a melamine and a soybean meal particle bound together collocated, a single melamine particle and a single soybean meal particle. These five arrangement statuses were spread on Teflon Spectralon surface artificially (Fig. 1) and measured with the NIRM imaging system.

Set B: Three mix soybean meal samples contaminated with $5 \%, 0.5 \%$ and $0.1 \%$ melamine were prepared in laboratory using three soybean meal samples and three melamine samples by a mixer (REAX 20/8; Heidolph, Schwabach, Germany). Each sample was spread on the Teflon Spectralon surface in a single layer and scanned. Scanning areas were $4025 \mu \mathrm{m} \times 4224 \mu \mathrm{m}, 4299 \mu \mathrm{m} \times 6825 \mu \mathrm{m}$ and $3549 \mu \mathrm{m} \times 6125 \mu \mathrm{m}$ corresponding to $5 \%, 0.5 \%$ and $0.1 \%$ melamine, respectively. 


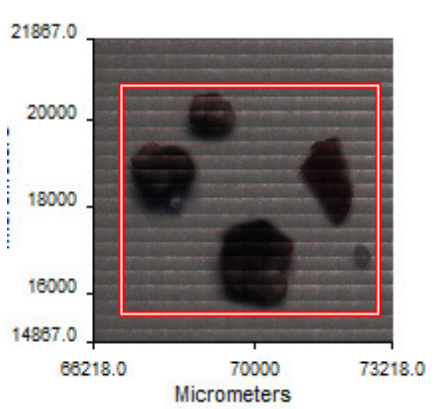

(a)

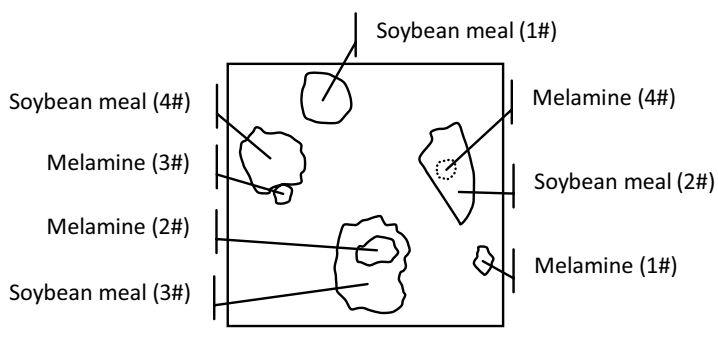

(b)

Fig. 1. Five arrangement statuses for the particles from soybean meal and melamine. (a) Visible light image. (b) Schematic of five arrangement statuses.

\subsection{Data analysis}

A key issue in NIRM imaging is how to extract relevant information from the huge amount of data.

Preprocessing. The main purpose of preprocessing is to remove the spectral and spatial artifacts, such as rough surfaces, optic effects, and detector noise, etc. To compare the effectiveness of different preprocessing for identifying melamine in soybean meal, the baseline offset correction, derivative and noise reduction methods were used separately or in combination.

Baseline offset correction: The slope of the baseline in a particular region is applied in Baseline offset correction to calculate the correction for all spectra in the image. To specify the Baseline range, a spectrum region with no significant absorption needs to be found.

Derivative: The first derivative removes any baseline offset, and the second derivative also removes any linear slope. The image was preprocessed by applying a derivative using the Savitzky-Golay algorithm.

Noise reduction: Principal Component Analysis (PCA) is applied to reduce noise in spectra. The PCA algorithm extracts the 20 most significant factors. The image is then reconstructed from extracted factors and displayed. By excluding those factors that are dominated by noise in this way, spectral noise is reduced in the reconstructed image. Unlike smoothing, the removal of noise factors does not lead to the broadening of the spectra peaks.

Extraction of Distribution Maps. Extraction has to be as accurate as possible to avoid pixel misclassification. Three different methods of extracting relevant information from scanned image data were used in this paper, which included two univariate analysis methods (image at a single wavelength and image at peak integration) and one multivariate analysis method (PCA, unsupervised classification).

In a single wavelength image, the ordinate value is the value (in absorbance) at a particular wavelength in each spectrum.

For a peak integration image, the ordinate value is calculated from one, two or three areas. At each pixel position, the area under specific spectral peaks is calculated. The resulting values form a new image in which higher pixel intensity reflects higher compound concentration.

The PCA analysis was to extract the eight most significant features in the spectra using a basic PCA. The data are projected on a new principal component subspace. The principal components are iteratively computed, the first being constructed to explain as components are known as scores. After the PCA transformation, each column of the score matrix is folded back to form an image that represents pixel variability along the corresponding components.

\section{Results and Discussion}

\subsection{Average absorbance imaging}

The image of average absorbance of sample set $A$ is shown in Fig. 2. The spectrum at pixel $(72,518$, $16,957)$ was the reference spectrum of melamine, and the spectrum at pixel $(68,994,20,181)$ was the reference spectrum of soybean meal. The spectral difference of melamine and soybean is significant, indicating the possibility to find a characteristic 

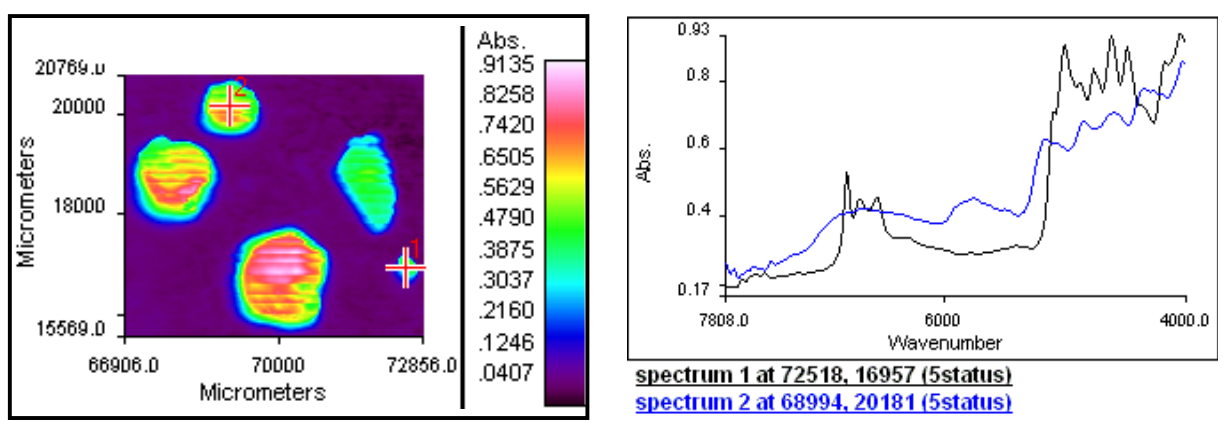

Fig. 2. The average absorbance imaging of sample A.

wavelength or area to identify the melamine present in the mixture of soybean meal and melamine.

For melamine, there was a very strong absorption from 6900 to $6450 \mathrm{~cm}^{-1}$, a region which has not been found in soybean meal spectra. Although there was abundant absorption information from 5100 to $4400 \mathrm{~cm}^{-1}$ in melamine spectra, there was also abundant absorption information in soybean meal spectra.

\subsection{Single wavelength imaging}

Image at a specific wavelength is the simplest method of obtaining distribution maps. Figure 3 gave the identifying result at a specific wavelength with different preprocessing methods. For image preprocessed by baseline offset correction, image at $6805 \mathrm{~cm}^{-1}$ can be applied to identify melamine $1 \#$, $2 \#, 3 \#$ and $4 \#$. Melamine $4 \#$ was under soybean meal particle, and the thickness of the soybean meal 2\# was about $150 \mu \mathrm{m}$.

The first- and second-order derivative of a spectrum usually has sharper features than the original spectra. However, the image preprocessed by first derivative at single wavelength $\left(6854 \mathrm{~cm}^{-1}\right)$ did not identify melamine $4 \#$ under soybean meal particle as good as the baseline offset correction. Secondderivative spectra have sharp minima where there are maxima in the original spectrum. Figure 3(c) showed similar result with Fig. 3(a). Noise reduction and baseline offset correction showed similar result of Fig. 3(a).

It can be seen from the above results that single wavelength imaging gave positive identification of melamine, which is the simplest method in revealing the localization of chemical compounds. In general, overlaps in complex systems may prevent identification of specific wavelength, especially in the NIR range. However, melamine is a triazine heterocyclic organic compound, and composed only with nitrogen heterocyclic ring and $-\mathrm{NH}_{2}$. Because $\mathrm{NH}_{2}$ has a special combination band in NIR range, NIR analysis has the advantages to detect $-\mathrm{NH}_{2}$. Melamine has three strong characteristic peaks in the range of $6900-6450 \mathrm{~cm}^{-1}$. However, soybean meal is a complex compound, and has a broader absorption band in the range of $6900-6450 \mathrm{~cm}^{-1} .6805 \mathrm{~cm}^{-1}$ is the $\mathrm{N}-\mathrm{H}$ combination band $(\nu \mathrm{N}-\mathrm{H}$ asymmetric and $\nu \mathrm{N}-\mathrm{H}$ symmetric combination) from primary amides. This wavelength is very sensitive for melamine and not sensitive for soybean meal.

\subsection{Peak integration imaging}

Figure 4 shows the image of peak integration with different preprocessing. All preprocessing methods gave similar results for melamine $1 \#, 2 \#$ and $3 \#$. The preprocessing method of first derivative showed the best identifying result for melamine $4 \#$.

Similar to single wavelength image analysis, peak integration under $6805 \mathrm{~cm}^{-1}$ is also sensitive for detecting melamine. It was seen from Fig. 4(b) that there was abundant absorption information from 5100 to $4400 \mathrm{~cm}^{-1}$ in melamine spectra, which maybe useful for detecting the melamine. However, there was also abundant absorption information in soybean meal spectra, which interferes with that of melamine. In Fig. 4(b), there was significant difference between melamine and soybean meal at the range of $5300-5000 \mathrm{~cm}^{-1}$. This study also tried to use the peak integration under this range to detect the melamine (Fig. 5). In Fig. 5(a) the detection of melamine was disturbed by information from soybean, which was not good for detecting 

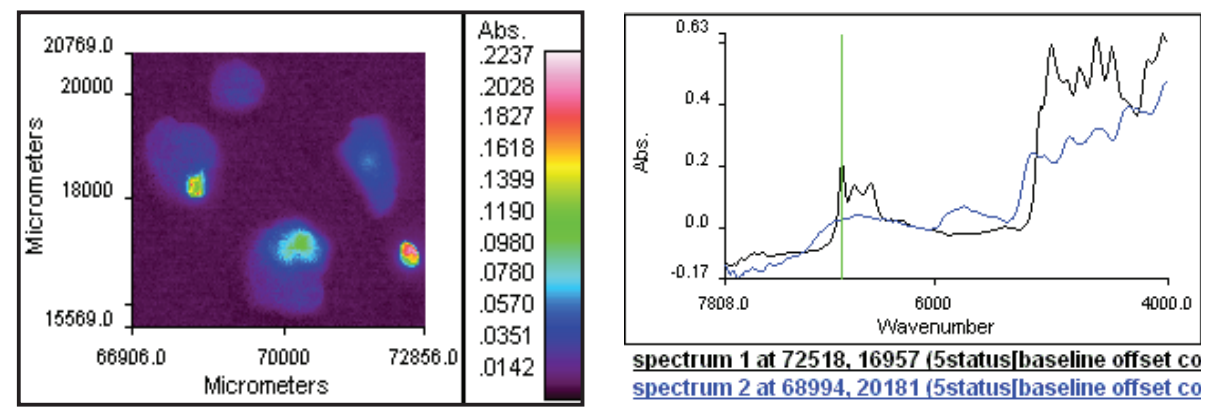

spectrum 1 at 72518, 16957 (5status[baseline offset co spectrum 2 at 68994,20181 (5status[baseline offset co

(a)
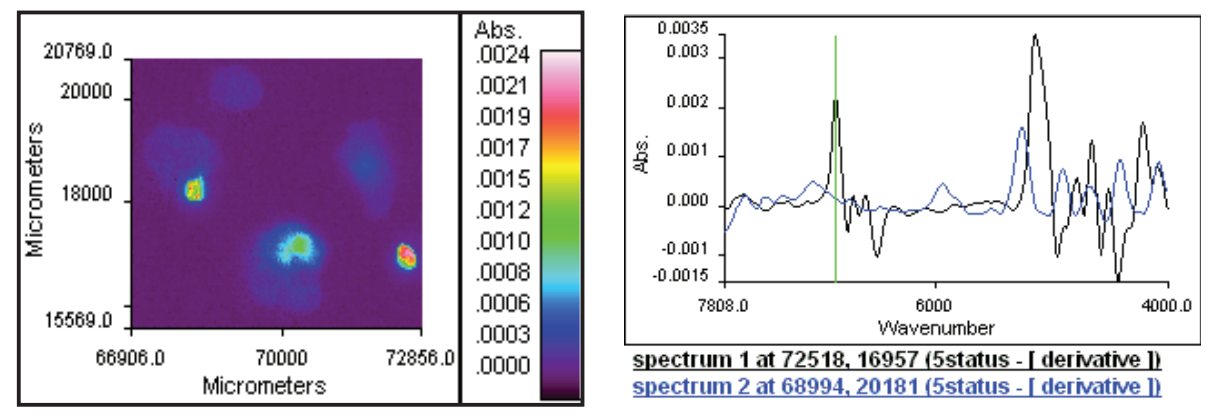

(b)
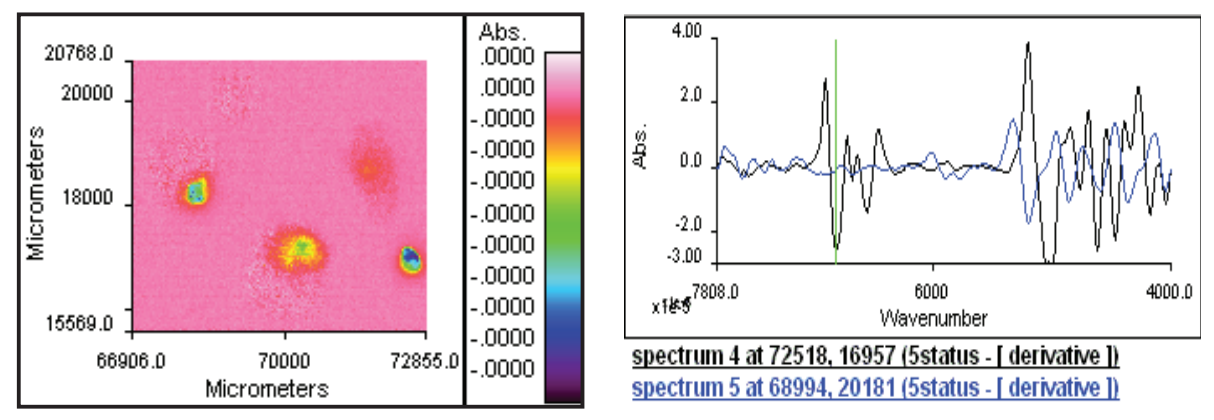

spectrum 4 at 72518,16957 (5status - [ derivative ]) spectrum 5 at 68994,20181 (5status - [ derivative ])

(c)
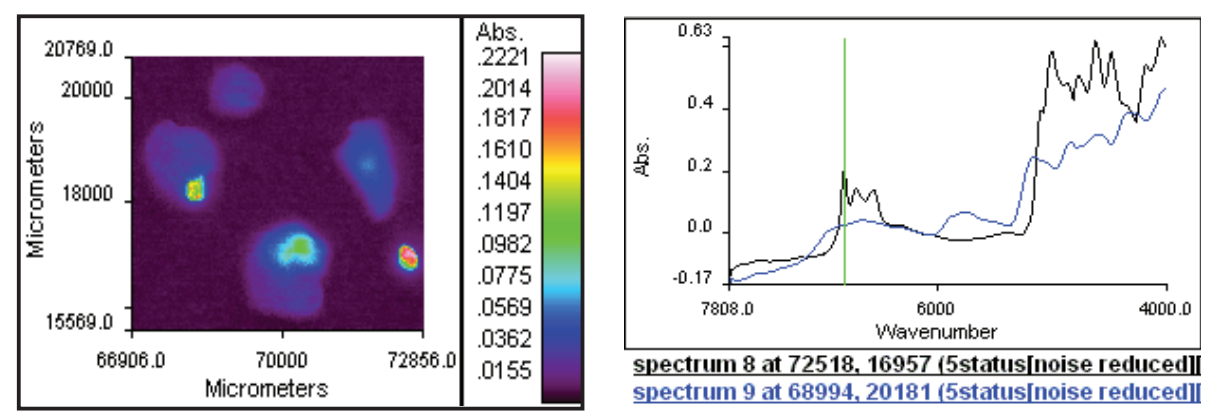

(d)

Fig. 3. Single wavelength images with different preprocessing. (a) Baseline offset correction (Single wavelength $=6805 \mathrm{~cm}^{-1}$ ). (b) First derivative (Single wavelength $\left.=6854 \mathrm{~cm}^{-1}\right)$. (c) Second derivative (Single wavelength $\left.=6805 \mathrm{~cm}^{-1}\right) .(\mathrm{d})$ Noise reduction and baseline offset correction (Single wavelength $=6805 \mathrm{~cm}^{-1}$ ). 


\section{Z. Yang et al.}
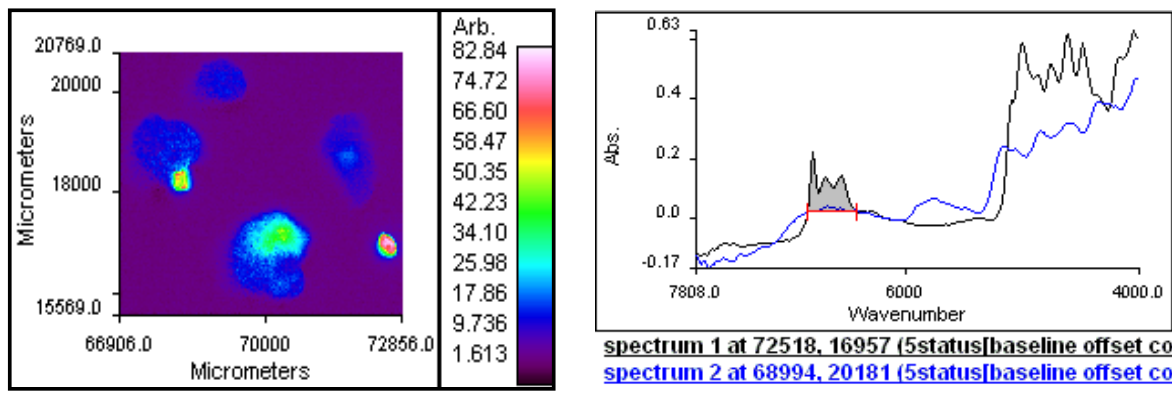

spectrum 1 at 72518,16957 (5status[baseline offset co spectrum 2 at 68994,20181 (5status bbaseline offset co

(a)
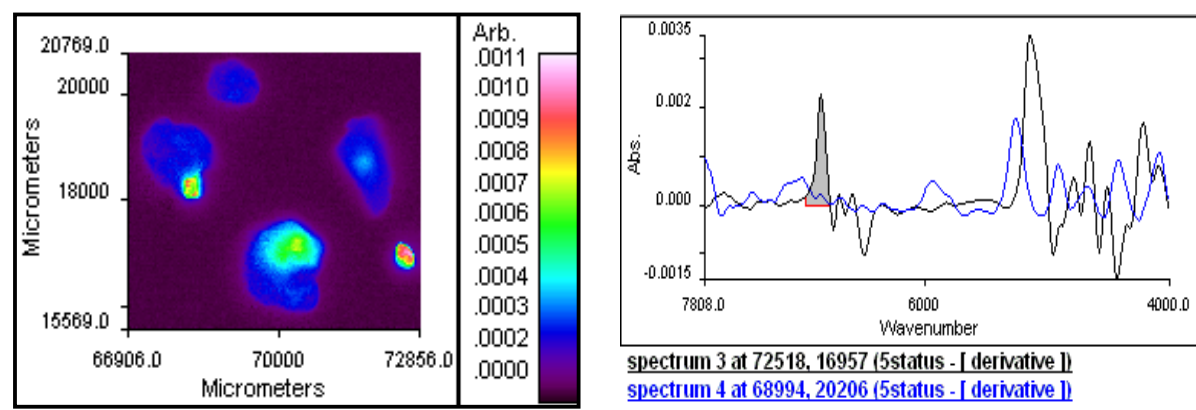

spectrum 3 at 72518.16957 (5status - [ derivative ]) spectrum 4 at 68994, 20206 (5status - [ derivative ]l)

(b)
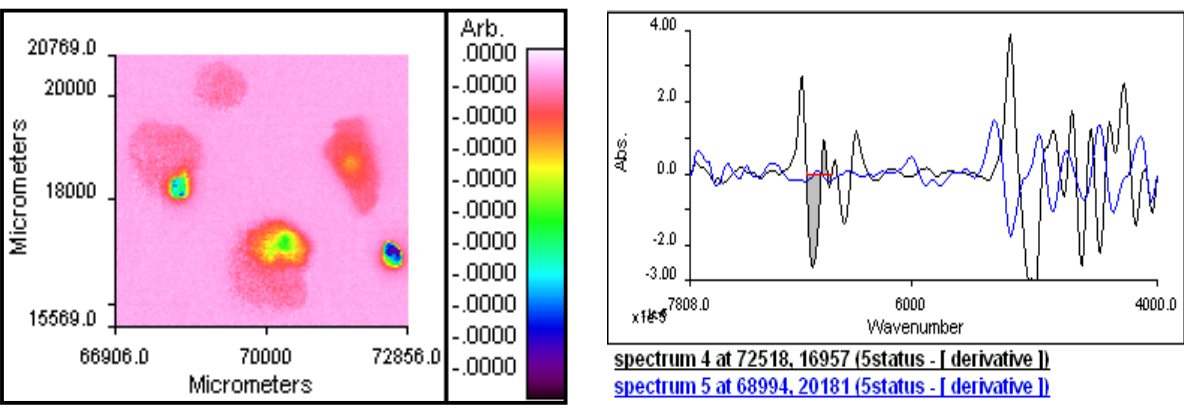

spectrum 4 at 72518,16957 (5status - [ derivative ] spectrum 5 at 68994,20181 (5status - [ derivative ])

(c)
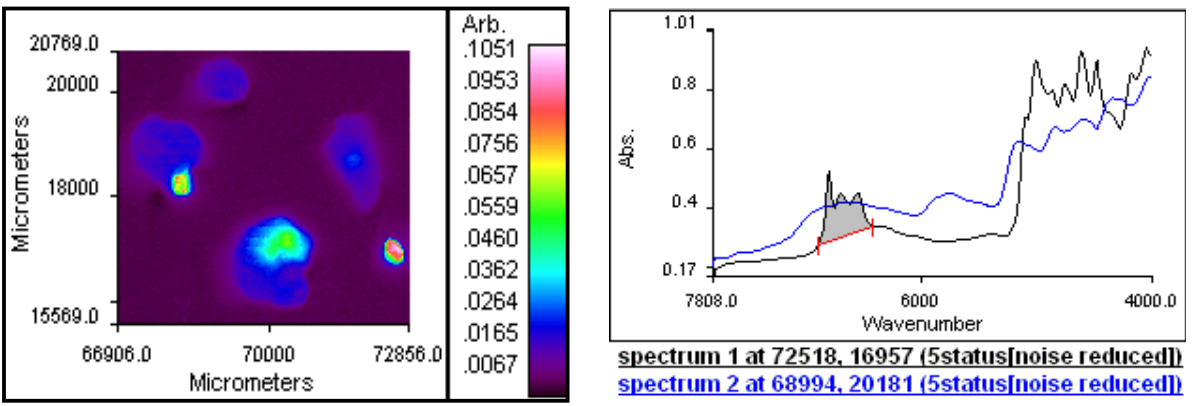

spectrum 1 at 72518,16957 (5status [noise reduced]) spectrum 2 at 68994,20181 (5status [noise reduced])

(d)

Fig. 4. Image under peak integration with different preprocessing. (a) Baseline offset correction (the area under $6846-6430 \mathrm{~cm}^{-1}$ with 2 base points). (b) First derivative (the area under $6980-6780 \mathrm{~cm}^{-1}$ without base points). (c) Second derivative (the area under $6860-6650 \mathrm{~cm}^{-1}$ without base points). (d) Noise reduction (the area under $6900-6430 \mathrm{~cm}^{-1}$ with 2 base points). 

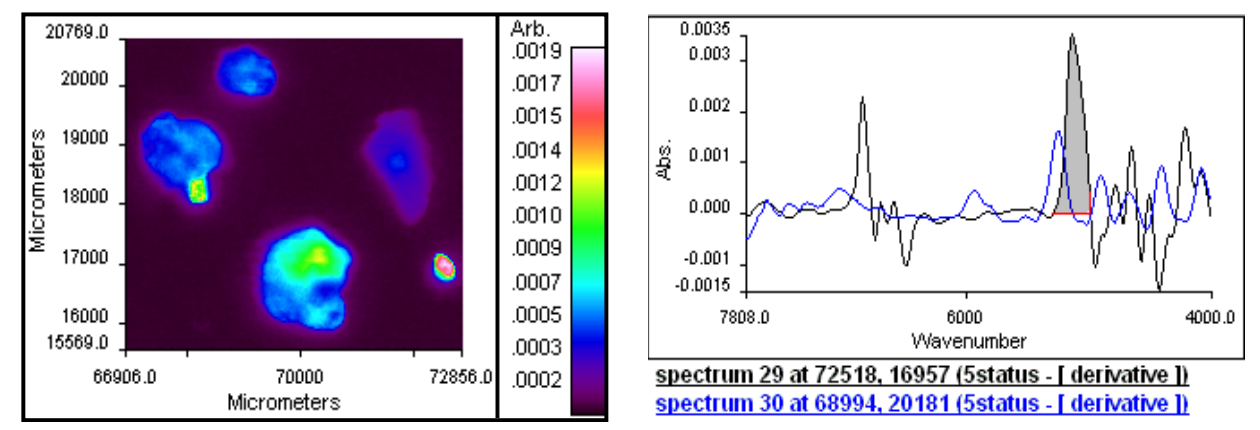

(a)
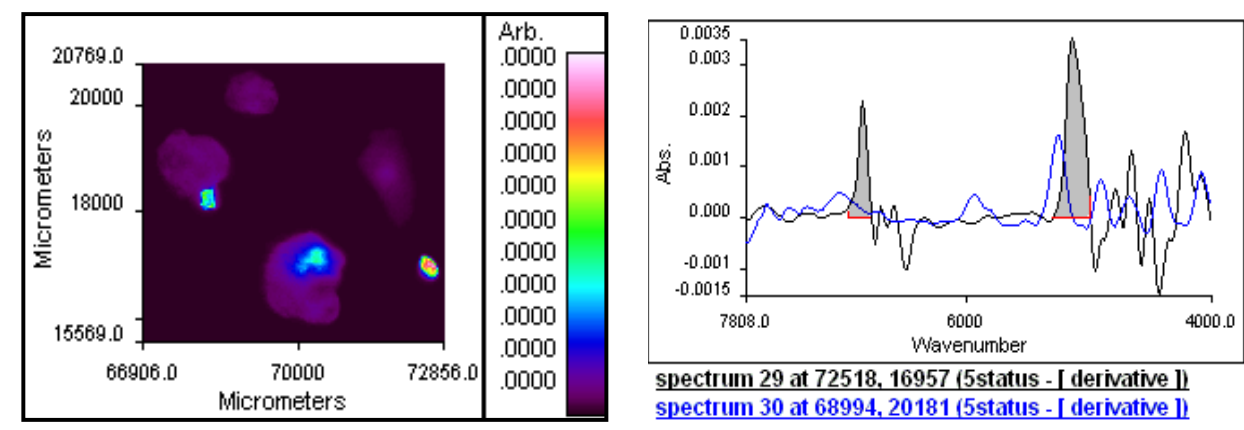

(b)

Fig. 5. Peak integration images with different wavelength range. (a) First derivative (the area under $5300-5000 \mathrm{~cm}^{-1}$ without base points). (b) First derivative (the area under $6980-6780 \mathrm{~cm}^{-1}$ and $5300-5000 \mathrm{~cm}^{-1}$ without base points).

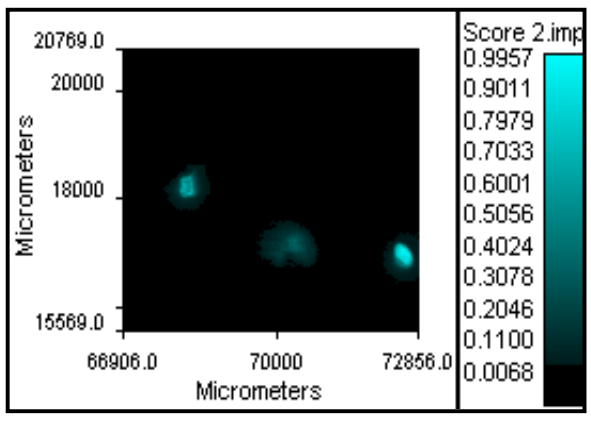

(a)

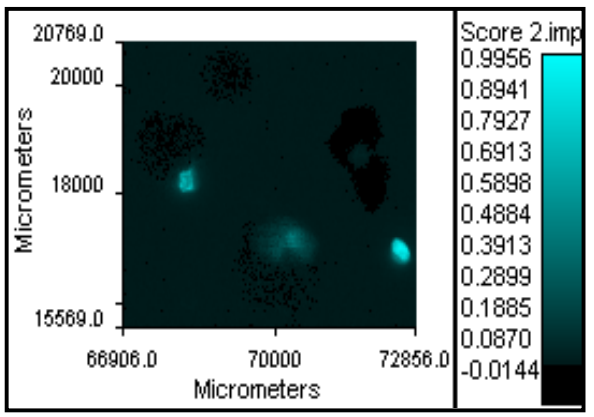

(c)

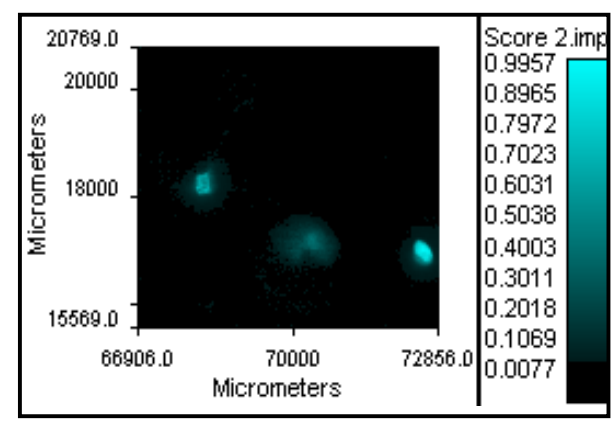

(b)

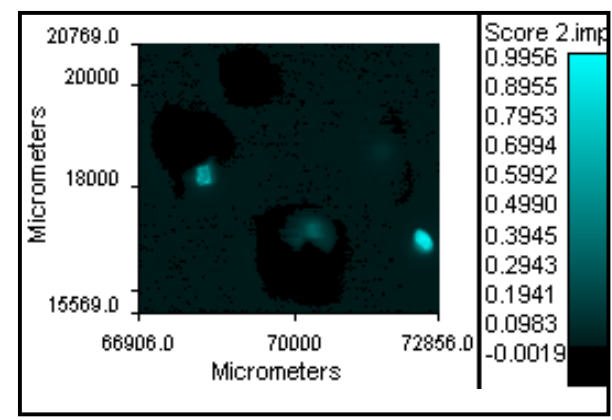

(d)

Fig. 6. Second score images of PCA analysis with different preprocessing. (a) Baseline offset correction. (b) First derivative. (c) Second derivative. (d) Noise reduction. 


\section{Z. Yang et al.}

melamine in $4 \#$. Combining $5300-5000 \mathrm{~cm}^{-1}$ and $6980-6780 \mathrm{~cm}^{-1}$, the peak integration image was shown in Fig. 5(b). It strengthened the signal intensity of pure melamine, but weakened the melamine information effected by soybean meal.

\subsection{PCA imaging}

With univariate analysis, all known ingredients need to be scanned to characterize their spectral signature. However, if the constituent is unknown, PCA analysis is more appropriate. PCA has been

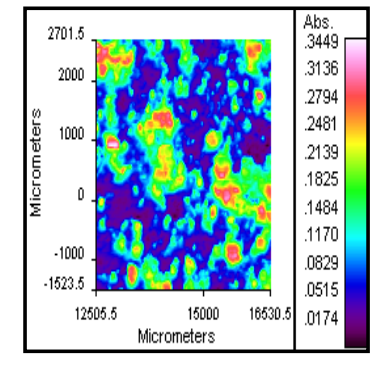

Average absorbance

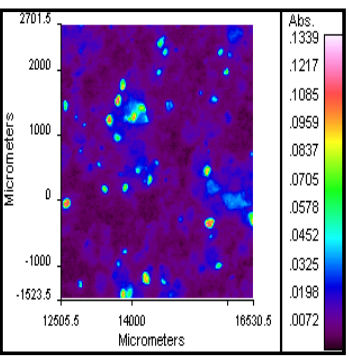

Single wavelength

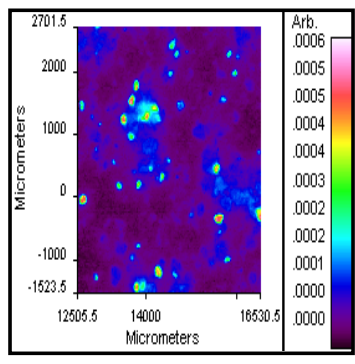

Peak integration

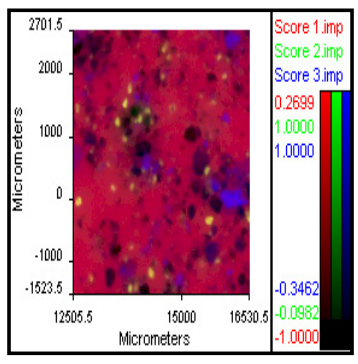

PCA analysis
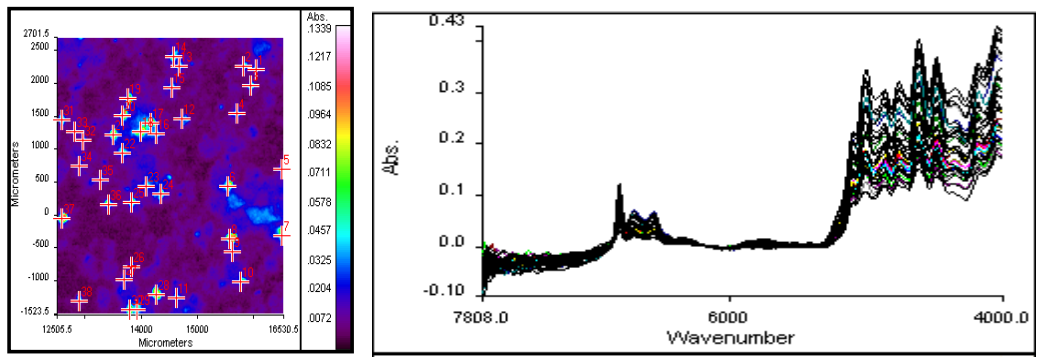

The spectra of particles identified as melamine

(a)

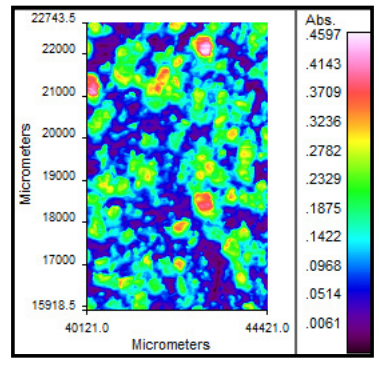

Average absorbance

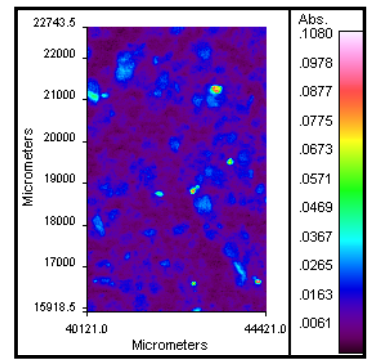

Single wavelength

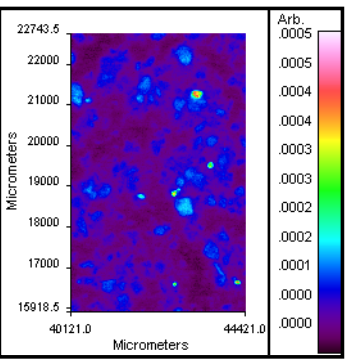

Peak integration

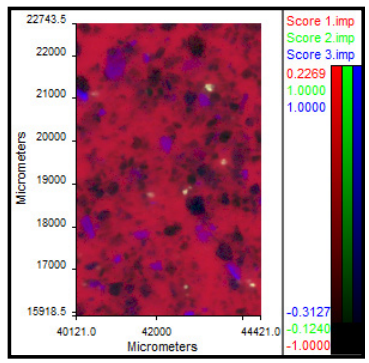

PCA analysis
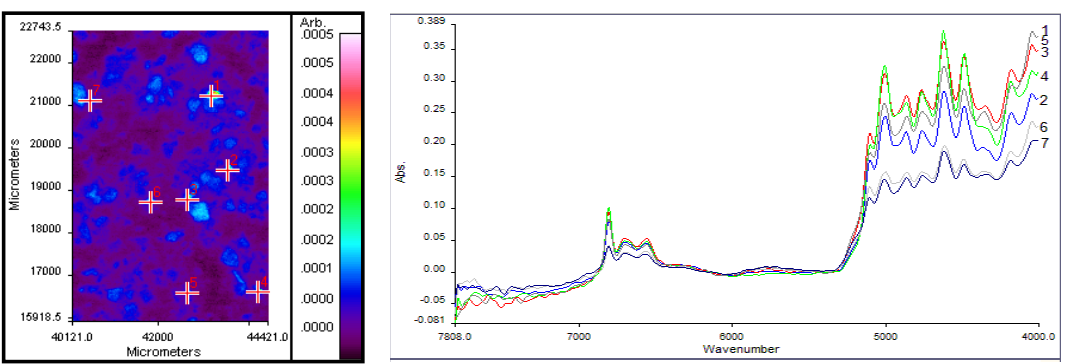

The spectra of particles identified as melamine

(b)

Fig. 7. The results of detecting melamine in samples from set B. (a) Real mixed soybean meal sample with $5 \%$ melamine. (b) Real mixed soybean meal sample with $0.5 \%$ melamine. (c) Real mixed soybean meal sample with $0.1 \%$ melamine. 


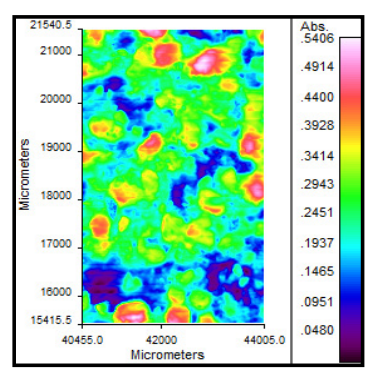

Average absorbance

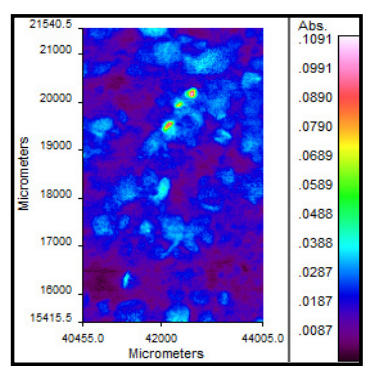

Single wavelength

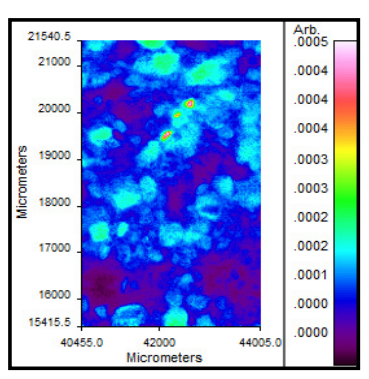

Peak integration

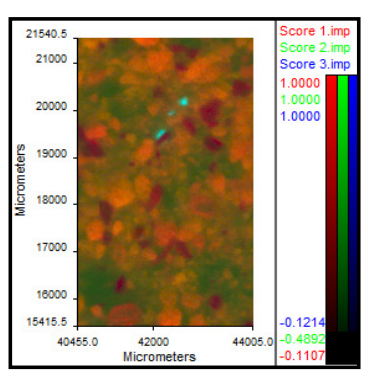

PCA analysis
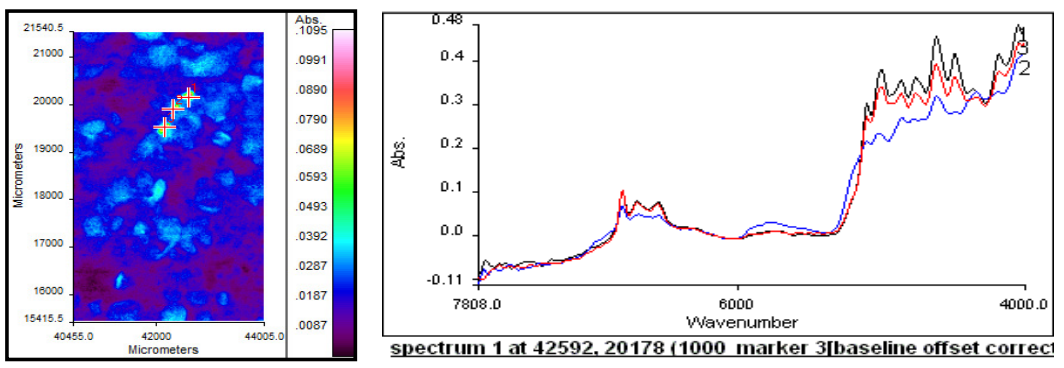

The spectra of particles identified as melamine

(c)

Fig. 7. (Continued)

extensively used in NIR image analysis to determine compound distribution. ${ }^{21}$ Information from melamine can be mainly extracted in 2nd score images. Figure 6 was 2nd score images of sample with different preprocessing. The preprocessing method of second derivative gave the best identifying result for melamine $4 \#$.

\subsection{Validation of methods}

Sample set B was used to verify the above proposed methods by sample set A. The results in Fig. 7 showed that melamine in the three mixture samples had been detected successfully, which indicates that NIRM imaging is a useful tool for detecting melamine in soybean meal.

Univariate analysis, such as single wavelength image and peak integration image, is the simplest method in revealing chemical localization in the scanned sample. The study reveals that $6805 \mathrm{~cm}^{-1}$ is very sensitive to melamine in soybean meal. However, when the constituent compound of the matrix material is unknown, PCA analysis is an effective method. NIRM imaging is potential technology for automatic feed inspection. The simple extracting method is very important and will hold a key role for this automatic inspection.

\section{Acknowledgments}

The project is funded by the European Commission under the Seventh Framework Programme (Quality and safety of feeds and food for Europe (QSAFFE), Contract No. FP7-KBBE-2010-4) and Program for New Century Excellent Talents in University (NCET-10-0785).

\section{References}

1. D. C. Pérez-Marín, A. Garrido-Varo, J. E. GuerreroGinel, A. Gómez-Cabrera, "Near-infrared reflectance spectroscopy (NIRS) for the mandatory labelling of compound feedingstuffs: Chemical composition and open-declaration," Anim. Feed Sci. Technol. 116, 333-349 (2004).

2. Z. Yang, L. Han, X. Liu, Q. Li, "Detecting and quantifying meat meal or meat and bone meal contamination in fishmeal by visible and near infrared reflectance spectra," Anim. Feed Sci. Technol. 147, 357-367 (2008).

3. F. Clarke, "Extracting process-related information from pharmaceutical dosage forms using near infrared microscopy," Vib. Spectrosc. 34, 25-35 (2004).

4. J. Dubois, J.-C. Wolff, J. K. Warrack, J. Schoppelrei, E. N. Lewis, "NIR chemical imaging for counterfeit pharmaceutical products analysis," Spectroscopy 22, 40-50 (2007). 
5. E. Lee, W. X. Huang, P. Chen, E. N. Lewis, R. V. Vivilecchia, "High-throughput analysis of pharmaceutical tablet content uniformity by near-infrared chemical imaging," Spectroscopy 21, 24-32 (2006).

6. E. N. Lewis, J. Schoppelrei, E. Lee, "Molecular spectroscopy workbench — Near-infrared chemical imaging and the PAT initiative," Spectroscopy 19, 26-36 (2004).

7. M. Lopes, J. Wolff, "Investigation into classification/sourcing of suspect counterfeit Heptodin TM tablets by near infrared chemical imaging," Anal. Chim. Acta 633, 149-155 (2009).

8. C. Ravn, E. Skibsted, R. Bro, "Near-infrared chemical imaging (NIR-CI) on pharmaceutical solid dosage forms - Comparing common calibration approaches," J. Pharm. Biomed. Anal. 48, 554-561 (2008).

9. V. Baeten, P. Dardenne, "Applications of nearinfrered imaging for monitoring agricultural food and feed products," Spectrochemical Analysis using Infrared Multichannel Detectors, R. Bhargava, I. W. Levin, Eds., pp. 283-300, Blackwell Publishing Ltd, Oxford, UK (2005).

10. H. F. G. Grahn, P. Geladi, Techniques and Applications of Hyperspectral Image Analysis, John Wiley \& Sons Ltd, Chichester, UK (2005).

11. J. A. Fernandez Pierna, V. Baeten, P. Dardenne, J. Dubois, E. N. Lewis, J. Burger, Spectroscopic imaging, Comprehensive Chemometrics: Chemical and Biochemical Data Analysis, S. D. Brown, R. Tauler, B. Walczak, Eds., Vol. 4, pp. 173-192, Elsevier, Amsterdam, Netherlands (2009).

12. D. Ariana, R. Lu, D. Guyer, "Near-infrared hyperspectral reflectance imaging for detection of bruises on pickling cucumbers," Comput. Electron. Agr. 53, 60-70 (2006).

13. J. Dubois, E. Neil Lewis, F. S. Fry, E. M. Calvey, "Bacterial identification by near-infrared chemical imaging of food-specific cards," Food Microbiol. 22, 577-583 (2005).

14. P. Williams, P. Geladi, G. Fox, M. Manley, "Maize kernel hardness classification by near infrared (NIR) hyperspectral imaging and multivariate data analysis," Anal. Chim. Acta 653, 121-130 (2009).

15. J. P. Wold, M. O'Farrell, M. Høy, J. Tschudi, "Online determination and control of fat content in batches of beef trimmings by NIR imaging spectroscopy," Meat Sci. 89, 317-324 (2011).
16. V. Baeten, C. von Holst, A. Garrido, J. Vancutsem, A. Michotte Renier, P. Dardenne, "Detection of banned meat and bone meal in feedstuffs by nearinfrared microscopic analysis of the dense sediment fraction," Anal. Bioanal. Chem. 382, 149-157 (2005).

17. B. Delarozadelgado, A. Soldado, A. Martinezfernandez, F. Vicente, A. Garridovaro, D. Perezmarin, M. Delahaba, J. Guerreroginel, "Application of near-infrared microscopy (NIRM) for the detection of meat and bone meals in animal feeds: A tool for food and feed safety," Food Chem. 105, 1164-1170 (2007).

18. J. A. Fernández Pierna, V. Baeten, P. Dardenne, "Screening of compound feeds using NIR hyperspectral data," Chemom. Intell. Lab. Syst. 84, 114118 (2006).

19. V. Fernandez-Ibanez, T. Fearn, A. Soldado, B. de la Roza-Delgado, "Spectral library validation to identify ingredients of compound feedingstuffs by near infrared reflectance microscopy," Talanta $\mathbf{8 0}$, 54-60 (2009).

20. Z. Yang, L. Han, J. A. F. Pierna, P. Dardenne, V. Baeten, "The potential of near infrared microscopy to detect, identify and quantify processed animal by-products," J. Near Infrared Spectrosc. 19, 211231 (2011).

21. C. Gendrin, Y. Roggo, C. Collet, "Pharmaceutical applications of vibrational chemical imaging and chemometrics: A review," J. Pharm. Biomed. Anal. 48, 533-553 (2008).

22. FEFAC, "FEFAC welcomes Commission decision to extend testing requirements on melamine to soybean products from China," Press review (2008).

23. F. Sun, W. Ma, L. Xu, Y. Zhu, L. Liu, C. Peng, L. Wang, H. Kuang, C. Xu, "Analytical methods and recent developments in the detection of melamine," TrAC, Trends Anal. Chem. 29, 1239-1249 (2010).

24. L. J. Mauer, A. A. Chernyshova, A. Hiatt, A. Deering, R. Davis, "Melamine detection in infant formula powder using near- and mid-infrared spectroscopy," J. Agric. Food Chem. 57, 3974-3980 (2009).

25. S. A. Haughey, S. F. Graham, E. Cancouët, C. T. Elliott, "The application of near-infrared reflectance spectroscopy (NIRS) to detect melamine adulteration of soya bean meal," Food Chem. 136, 15571561 (2013). 